\title{
Single-cell analysis of skin immune cells reveals an Angptl4- ifi20b axis that regulates monocyte differentiation during
} wound healing

Wei Kiat Jonathan Wee ${ }^{1,2}$, Zun Siong Low ${ }^{1}$, Chin Kiat Ooi ${ }^{2}$, Benjamin Patrana Henategala ${ }^{2}$, Zhi Guang Ridley Lim², Yun Sheng Yip ${ }^{1}$, Marcus Ivan Gerard Vos ${ }^{1}$, William Wei Ren $\operatorname{Tan}^{1}$, Hong Sheng Cheng (iD ${ }^{1}$ and Nguan Soon Tan (iD) ${ }^{1,2 凶}$

(C) The Author(s) 2022

The persistent inflammatory response at the wound site is a cardinal feature of nonhealing wounds. Prolonged neutrophil presence in the wound site due to failed clearance by reduced monocyte-derived macrophages delays the transition from the inflammatory to the proliferative phase of wound healing. Angiopoietin-like 4 protein (Angptl4) is a matricellular protein that has been implicated in many inflammatory diseases. However, its precise role in the immune cell response during wound healing remains unclear. Therefore, we performed flow cytometry and single-cell RNA sequencing to examine the immune cell landscape of excisional wounds from Angptl $4^{+/+}$and Angptl $4^{-/-}$mice. Chemotactic immune cell recruitment and infiltration were not compromised due to Angptl4 deficiency. However, as wound healing progresses, Angpt/4 ${ }^{-1-}$ wounds have a prolonged neutrophil presence and fewer monocyte-derived macrophages than Angpt/4 $4^{+/+}$and Angpt/4 $4^{\mathrm{LySM}-/-}$ wounds. The underlying mechanism involves a novel Angptl4-interferon activated gene 202B (ifi202b) axis that regulates monocyte differentiation to macrophages, coordinating neutrophil removal and inflammation resolution. An unbiased kinase inhibitor screen revealed an Angpt/4-mediated kinome signaling network involving S6K, JAK, and CDK, among others, that modulates the expression of ifi202b. Silencing ifi202b in Angpt/4 ${ }^{-/-}$monocytes, whose endogenous expression was elevated, rescued the impaired monocyte-to-macrophage transition in the in vitro reconstituted wound microenvironment using wound exudate. GSEA and IPA functional analyses revealed that ifi202bassociated canonical pathways and functions involved in the inflammatory response and monocyte cell fate were enriched. Together, we identified ifi202b as a key gatekeeper of monocyte differentiation. By modulating ifi202b expression, Angptl 4 orchestrates the inflammatory state, innate immune landscape, and wound healing process.

Cell Death and Disease (2022)13:180; https://doi.org/10.1038/s41419-022-04638-7

\section{INTRODUCTION}

Poor healing wounds are a silent epidemic affecting a large fraction of the world population, often overshadowed by comorbid conditions such as diabetes [1]. The biomedical and socioeconomic burdens are expected to worsen as the population ages. Patients with poor healing wounds frequently find themselves forced to choose between their work commitments and compliance with medical management. Others may be permanently impaired from performing their occupational job. Thus, much attention and resources are needed to understand the biological mechanisms underlying cutaneous wound complications.

Normal wound healing consists of a finely tuned pattern of integrated biological events that include hemostasis, inflammation, proliferative, and maturation phases to re-establish a new epithelial barrier [2]. Inflammation plays both positive and negative roles in wound healing - the level and length of inflammation dictate the healing time and quality of the repair. Neutrophils are among the earliest immune cells recruited to the wound site [3]. While neutrophils play important roles in the early inflammatory phase of the wound, their timely apoptosis, followed by uptake by phagocytic macrophages, is a key event necessary for inactivation and departure from the inflammatory phase of wound repair [4]. However, chronic wounds are in pathologic inflammation due to a postponed, incomplete, or uncoordinated healing process [5]. Cell-cell and cell-matrix communication, which coordinates the essential and complex inflammatory response during normal wound healing, is disrupted in chronic wounds. This synchrony is mediated by the action of central players, such as chemokines and matricellular proteins [6]. Matricellular proteins can associate with diverse proteins of the extracellular matrix, bridging them with cognate cell surface receptors and contributing to paracrine communication during wound healing [7]. However, there is still much to learn about the dynamics of cell-matrix communication that modulate inflammation in acute skin wounds and how it becomes deranged to generate chronic wounds.

\footnotetext{
${ }^{1}$ Lee Kong Chian School of Medicine, Nanyang Technological University Singapore, 11 Mandalay Road, Singapore 308232, Singapore. ${ }^{2}$ School of Biological Sciences, Nanyang Technological University Singapore, 60 Nanyang Drive, Singapore 637551, Singapore. ${ }^{凶}$ email: nstan@ntu.edu.sg
}

Edited by Professor Eleonora Candi

Received: 1 December 2021 Revised: 24 January 2022 Accepted: 7 February 2022

Published online: 24 February 2022 
Angiopoietin-like 4 protein (Angptl4) is a secreted matricellular protein that undergoes proteolytic cleavage, releasing an $\mathrm{N}$-terminal coiled-coil domain (nAngpt|4) and a C-terminal fibrinogen-like domain (cAngpt/4). nAngpt/4 binds to lipoprotein lipase and inhibits its activity to regulate peripheral triglyceride metabolism [8]. cAngpt/4 interacts with specific integrins, cadherins, and ECM proteins to enhance their signaling $[9,10]$. Angptl4 has been implicated in modulating immune responses in various inflammatory diseases. Hematopoietic Angptl4 deficiency in hyperlipidemic mice causes leukocytosis [11]. Angptl4-deficient (Angpt $14^{-1-}$ ) mice were shown to have large numbers of macrophages in the spleen, and Angpt/4 $4^{-1-}$ macrophages produced large amounts of TNF- $a$ and inducible nitric oxide synthase due to abnormal fatty acid metabolism [12]. It was also shown that dietary fatty acids activate PPAR $\beta / \delta$ signaling in the bone marrow to negatively regulate mobilization, in part via Angptl4 production. The treatment of wild-type (Angptl $4^{+/+}$) mice with anti-cAngpt/4 antibody enhanced mobilization [13]. Despite its involvement in many inflammatory diseases, the precise role of Angptl4 in immune cells and responses remains unclear.

Angptl4 participates in many events that are important for proper wound healing. Upon wound injury, hyperproliferative wound keratinocytes secrete a large amount of cAngptl4 that acts as a coordinating conduit with other wound cell types [14]. cAngptl4 facilitates wound angiogenesis, accelerates keratinocyte and fibroblast migration, and reduces scar collagen deposition [15]. Wound healing in Angptl4 $4^{-1-}$ mice is delayed and shares many similarities with diabetic wounds. Wounds are associated with stalled inflammation, poor wound-related angiogenesis, and ruined ECM. Indeed, human and mouse diabetic wounds produce little cAngptl4 compared with nondiabetic wounds. The topical application of recombinant cAngptl4 accelerates wound closure in diabetic mice [16]. However, how Angpt/4 regulates cell-matrix communication to affect the immune cell response to injury is unknown. A better understanding will contribute to translatable therapies for chronic wounds and many other inflammatory diseases.

This study examines the immune cell landscape of excisional wounds from wild-type (Angptl $4^{+/+}$) and Angptl $4^{-/-}$mice. We uncovered that Angpt/4 deficiency in the wound site reduces monocyte-derived macrophages and prolongs the inflammatory phase of wound healing. In addition, Angpt/4 regulates the expression of interferon-activated gene 202B (ifi202b), which affects gene networks involved in monocyte cell fate.

\section{RESULTS \\ Angptl4 deficiency portends prolonged inflammation in wounds}

We first examined the temporal changes in immune subpopulations using a multicolor flow cytometry panel for CD11b (myeloid cell marker), Ly6G (neutrophil marker), Ly6C (mouse monocyte marker), and MHC-II (macrophage marker). Figure S1A shows representative plots outlining the gating strategy. Four subpopulations of monocytes and macrophages were observed in our excisional wound model - namely, Ly6C ${ }^{\text {hi }}$ MHC-II ${ }^{-v e}$ inflammatory monocytes, Ly6C ${ }^{\text {int }} \mathrm{MHC}-\mathrm{II}^{-\mathrm{ve}}$ monocytes, and Ly6C $\mathrm{C}^{+\mathrm{ve}} \mathrm{MHC}-\mathrm{II}^{+\mathrm{ve}}$

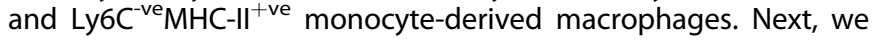
examined the changes in these immune cell subpopulations during wound healing in Angpt $14^{+/+}$and Angpt/ $4^{-/-}$mice. At 1 day postwounding (dpw), no significant difference in infiltrated neutrophils, inflammatory monocytes, and Ly6C ${ }^{+\mathrm{ve}} \mathrm{MHC}-\mathrm{II}^{+\mathrm{ve}}$ monocyte-derived macrophages was observed between Angptl $4^{+/+}$and Angpt/4 ${ }^{-/-}$wounds (Table 1, Fig. S1B). Interestingly, Angpt $14^{-1-}$ wounds had a higher percentage of infiltrated monocytes at 1 to $3 \mathrm{dpw}$ but a reduced percentage of mature Ly6C $^{-v e} \mathrm{MHC}-\mathrm{II}^{+\mathrm{ve}}$ macrophages at 1 to $5 \mathrm{dpw}$ compared with Angpt $14^{+/+}$wounds. Consistent with a role for macrophages in the 

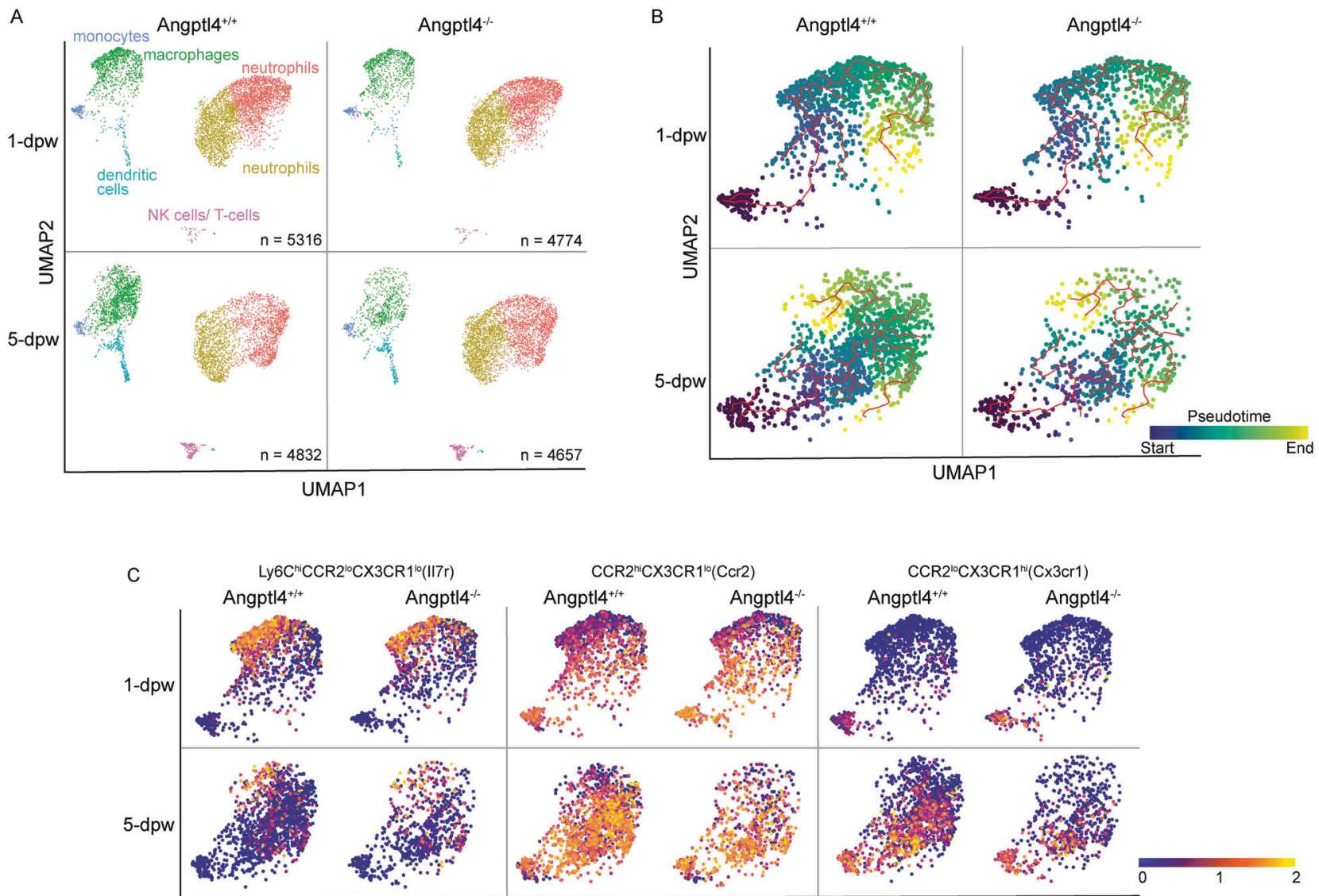

Fig. 1 Single-cell immune landscape during mouse skin excisional wound healing. A Individual UMAP dimensionality reduction plots of sequenced $\mathrm{CD}_{4} 5^{+}$immune cells from Angpt $4^{+/+}$and Angptl $4^{-1-}$ wounds at 1 and 5 days post-wounding (dpw). B UMAP dimension reduction plots showing cells from individual scRNA-seq samples ordered by pseudotime (color scale). Monocytes were marked as the start of pseudotime. Calculated cell trajectories are shown as red lines. C The distribution of cells expressing II $7 \mathrm{r}, \mathrm{Ccr} 2$ and $\mathrm{Cx} 3 \mathrm{cr} 1 \mathrm{during}$ the recovery of Angptl $4^{+/+}$and Angptl $4^{-/-}$wounds.

clearance of neutrophils, Angptl $4^{-1-}$ wounds had prolonged neutrophil persistence at 5- to 7-dpw. There were fewer Ly6C ${ }^{\mathrm{ve}} \mathrm{MHC}-\mathrm{II}^{+\mathrm{ve}}$ mature monocyte-derived macrophages in Angptl ${ }^{-1}$ - than Angptl $4^{+/+}$wounds across all examined time timepoints (Table 1). Following the peak of $\mathrm{Ly} 6 \mathrm{C}^{+\mathrm{ve}} \mathrm{MHC}-\mathrm{II}^{+\mathrm{ve}}$ and $\mathrm{Ly} \mathrm{CC}$ ${ }^{\mathrm{ve}} \mathrm{MHC}-\mathrm{II}^{+\mathrm{ve}}$ monocyte-derived macrophages at $3 \mathrm{dpw}$, their percentage decreased from 5- to 7-dpw in Angpt/4 ${ }^{+/+}$wounds $(59-77 \%)$. In contrast, their percentage increased in Angpt/4 ${ }^{-/-}$ wounds (28-50\%) (Table 1). Cytokine profiling of early wound exudates at $8 \mathrm{~h}$ and $1 \mathrm{dpw}$ from Angpt/4 $4^{+/+}$and Angpt $14^{-/-}$ wounds showed no significant difference in the examined 31 cytokines (Fig. S2A). An in vitro chemotaxis assay using bone marrow-derived monocytes (BMDMs) from Angptl $4^{+{ }^{+}}$and Angpt $14^{-1-}$ mice also showed no difference in invasiveness, suggesting that chemotactic immune cell recruitment and infiltration were not compromised due to Angpt/4 deficiency (Fig. S2B). Finally, we also examined neutrophils and macrophages in wounds of Angpt/4 $4^{\text {LysM-I- }}$ myeloid cell-specific Angptl4 deletion. Similar numbers of neutrophils and $\mathrm{Ly} \mathrm{C}^{-\mathrm{ve}} \mathrm{MHC}-\mathrm{II}^{+\mathrm{ve}}$ mature macrophages were found in Angpt/ $4^{\text {LysM-l- }}$ and Angpt $14^{\mathrm{f} / \mathrm{f}}$ wounds (Fig. S2C). Hence, Angptl4 deficiency affects cell-matrix interactions that potentially regulate monocyte to macrophage differentiation and portends prolonged inflammation during wound healing.

\section{Angptl4 deficiency results in impaired monocyte differentiation during wound healing}

Guided by our flow cytometry findings, single-cell RNA sequencing (scRNA-seq) was performed on sorted $\mathrm{CD} 45^{+}$cells from wound biopsies of Angpt/4 $4^{+/+}$and Angpt/4 $4^{-/-}$mice at 1 and 5 dpw. Cell sorting procedures were optimized, and 19,579 sequenced cells that met the quality control metrics were analyzed (Fig. S3). Six major immune cell clusters were identified from the aggregated scRNA-seq data (Fig. S4A). Probing the top 25 significantly enriched genes from each cluster against the ImmGen database [17] showed a close correlation with datasets from specific immune cell types, confirming the accurate annotation of the cell clusters (Fig. S4B). Thus, acute excisional skin wounds comprise mostly myeloid cells (neutrophils, monocytes, macrophages, and dendritic cells), with minor NK and T subpopulations.

Two clusters of neutrophils were identified from cluster annotation and analysis of the differentially expressed genes (DEGs) (Fig. S5A). Cluster 0 neutrophils expressed many protumorigenic factors, such as Cxcr2, TGF- $\beta 1$, Mmp9, and Lrg1. Cluster 1 neutrophils expressed various proinflammatory and proangiogenic factors, such as VEGF-A, TNF-a, and $\mathrm{Ccl} 3$ (Fig.S5A). In Angptl $4^{+/+}$wounds, infiltrated neutrophils (clusters 0 and 1) decreased by $17.5 \%$ from 1 to $5 \mathrm{dpw}$. In contrast, the decrease was only $2.3 \%$ in $\mathrm{Angptl}^{-1-}$ wounds, consistent with neutrophil persistence (Fig. 1A, Table 2).

The monocyte cluster was highly enriched in Ly6c2 expression, whereas monocyte-derived macrophages expressed high levels of MHC-II (H2-Aa) and Adgre1, or F4/80, a common macrophage marker. Moderate to low expression of Ly6c2 was enriched in the macrophage cluster, likely representing monocytes differentiating into macrophages (Fig. S5B). Very few cells expressed Cd207 and Cd169, biomarkers of Langerhans cells and dermal resident 
Table 2. Immune cell subpopulations in Angptl/4 $4^{+/+}$and Angpt/4 ${ }^{-/-}$wounds by scRNA-seq.

\begin{tabular}{|c|c|c|c|c|c|}
\hline \multirow[b]{2}{*}{ Cluster } & \multirow{2}{*}{$\begin{array}{l}\% \text { of infiltrated } \mathrm{CD}^{+} 5^{+} \text {cells } \\
\text { Cell type }\end{array}$} & \multirow{2}{*}{$\begin{array}{l}\text { 1-dpw } \\
\text { Angptl4 }^{+/+}\end{array}$} & \multicolumn{3}{|c|}{ 5-dpw } \\
\hline & & & Angptl4 ${ }^{-I-}$ & Angptl1 ${ }^{+/+}$ & Angptl14 ${ }^{-1-}$ \\
\hline 0 & neutrophils & 39.95 & 42.73 & 30.11 & 39.49 \\
\hline 2 & macrophages & 21.61 & 18.06 & 30.69 & 13.64 \\
\hline 3 & dendritic cells & 1.90 & 1.76 & 7.16 & 4.49 \\
\hline 4 & monocytes & 2.73 & 2.09 & 2.30 & 2.30 \\
\hline
\end{tabular}

The number of cells in each cluster was expressed as a percentage of the total number of $\mathrm{CD}_{4} 5^{+}$cells for each sample.

Table 3. Distribution of monocytes and monocyte-derived cells in Angpt $14^{+/+}$and Angpt/4 ${ }^{-/-}$wounds.

\begin{tabular}{|c|c|c|c|c|}
\hline \multirow[b]{2}{*}{ Monocytes/monocyte-derived macrophages } & \multicolumn{2}{|l|}{ 1-dpw } & \multicolumn{2}{|l|}{ 5-dpw } \\
\hline & Angptl $4^{+/+}$ & Angptl $4^{-1-}$ & Angptl4 $^{+/+}$ & Angptl14 ${ }^{-1-}$ \\
\hline Ly $_{6 C}$ hi $^{\text {CCR } 2}{ }^{\text {lo }} \mathrm{CX} 3 \mathrm{CR} 1^{\text {lo }}$ & 5.41 & 3.75 & 1.34 & 0.92 \\
\hline $\mathrm{CCR} 2^{\mathrm{lo}} \mathrm{C} \times 3 \mathrm{CR} 1^{\mathrm{hi}}$ & 0.23 & 0.30 & 3.76 & 0.81 \\
\hline
\end{tabular}

The numbers of each of these cell types are expressed as a percentage of the total cells passing QC for that sample, representing CD45 immune cells. Gene expression values are expressed based on the normalized, log-transformed, and scaled count values.

macrophages at $1 \mathrm{dpw}$, respectively [18] (Fig. S5C). No difference in infiltrated monocytes was observed between the two genotypes. The percentage of monocyte-derived macrophages increased by $9.1 \%$ in Angptl $4^{+/+}$wounds, whereas the percentage decreased in Angptl $4^{-1-}$ wounds from 1 to $5 \mathrm{dpw}$ (Table 2). Trajectory analysis revealed that monocytes have two main developmental branches (Fig. 1B), indicative of early and late wound immune responses [19]. Angptl4 deficiency resulted in reduced numbers of Ly6C ${ }^{\text {hi } C C R 2}{ }^{\text {lo }}{ }^{2 X} 3 C R 1^{\text {lo }}(\mathrm{II} 7 r>1)$ inflammatory monocytes, CCR2 ${ }^{\text {hi }} \mathrm{CX} 3 \mathrm{CR} 1^{\text {lo }}(\mathrm{Ccr} 2$, Adgre $1>1$ \& Ly $6 \mathrm{c} 2<1)$ and $\mathrm{CCR} 2{ }^{\mathrm{lo}} \mathrm{CX} 3 \mathrm{CR} 1^{\mathrm{hi}} \quad(\mathrm{Cx} 3 \mathrm{Cr} 1$, Adgre $1>1$ \& Ly $6 \mathrm{c} 2<1)$ monocytederived macrophages, with the most significant deficit in the number $(>50 \%)$ of monocyte-derived macrophages (Fig. 1C, Table 3). There was no significant difference in dendritic cells at $1 \mathrm{dpw}$ between the two genotypes; however, the percentage was lower at $5 \mathrm{dpw}$ in Angpt/4 $4^{-/-}$wounds than in Angpt/4 $4^{+/+}$wounds (Fig. 1A, Table 2), suggesting delayed wound closure in Angptl $4^{-1}$ mice. Dendritic cells are important to regain homeostasis upon tissue injury [20].

The dynamics between neutrophils and macrophages are important in the transition from inflammation to the proliferative phase of wound healing. Monocyte-derived macrophage numbers peak during the inflammatory phase and before declining during tissue formation [21]. Infiltrated neutrophils undergo apoptosis and are phagocytosed by macrophages. This dynamic is affected by Angptl4 deficiency. Our analyses revealed prolonged neutrophil persistence with markedly reduced mature macrophages in Angpt $4^{-1-}$ mice, which arose from impaired monocyte to macrophage differentiation. It also points to an impaired transition from inflammation to the proliferative phase of wound healing in Angptl $4^{-1-}$ wounds.

\section{Angptl4 regulates the expression of interferon-activated gene $202 \mathrm{~b}$ via S6K, FAK, and CDK signaling networks}

Our examination of the DEGs revealed that the expression of heme-binding protein 1 (Hebp1) and interferon activated gene 202B (ifi202b) was consistently elevated in 1- and 5-dpw Angpt $14^{-/-}$compared with Angptl $4^{+/+}$monocytes and macrophages, but not in other immune cell types (Table S1). While the expression of Hebp1 was higher than ifi202b in monocytes, its expression level was unchanged between monocytes and macrophages. Only the expression of ifi202b was further increased from monocytes to macrophages, overtaking Hebp1 and thus suggesting a potential role in monocyte to macrophage differentiation. We further confirmed the increased mRNA and protein expression of ifi202b in Angpt/4 ${ }^{-/-}$BMDMs (Fig. 2A).

Little information is known about the transcriptional regulation of the ifi202b gene. Thus, we first identified the regulatory sites in the mouse Ifi202b gene. Three DNAse I hypersensitive sites (DHS 1 -3 ) are potential regulatory sites in ifi202b, as suggested by data from the ENCODE database [22] (Fig. S6). All DHS 1-3 have increased susceptibility to DNAse I in Angpt/4 ${ }^{-1-}$ BMDMs compared with Angpt/4 ${ }^{+/+}$BMDMs, indicating increased chromatin accessibility (Fig. 2B). In silico analysis of DHS 1-3 identified putative binding sites for Jun, Fos, and Stat3. ChIP analysis confirmed the increased occupancy of these transcription factors at DHS $1-3$ in Angpt/4 ${ }^{-/-}$BMDMs compared with Angpt/4 ${ }^{+/+}$ BMDMs (Fig. 2C). Next, we performed an unbiased 133-kinase inhibitor screen using BMDMs to decipher signaling pathways responsible for Angptl4-mediated regulation of ifi202b expression. Kinase inhibitors that decrease the elevated expression of ifi202b in Angpt $14^{-1-}$ BMDMs indicate that their targets are involved in the signaling cascade. Similarly, kinase inhibitors that upregulate ifi202b expression in Angpt $14^{+/+}$BMDMs indicate that their targets suppress ifi202b expression (Fig. S7). We identified three kinases that significantly down- and upregulated the expression of ifi202b in Angpt/4 ${ }^{-/-}$and Angpt/4 ${ }^{+/+}$BMDMs, respectively (Fig. 2D). In the presence of Angptl4, we observed high S6K, FAK, and CDK activation that suppressed the expression of ifi202b. We also identified increased phospho-activated Jak1/2/3, Mek1/2, and Syk kinases in Angptl4 $4^{-1-}$ BMDMs that activate Jun, Fos, and Stat3 transcription factors (Fig. 2E). Integrating these data with protein interaction networks from the STRING database, we revealed the Angpt|4-mediated regulatory networks of the ifi202 gene in monocytes (Fig. 2F).

\section{Ifi202b regulates monocyte differentiation to macrophages} In the wound milieu, cell fate is guided by microenvironmental cues. For wounds, much of this cue is captured in the wound fluid [23]. Therefore, monocytes isolated from the peripheral blood and 
A

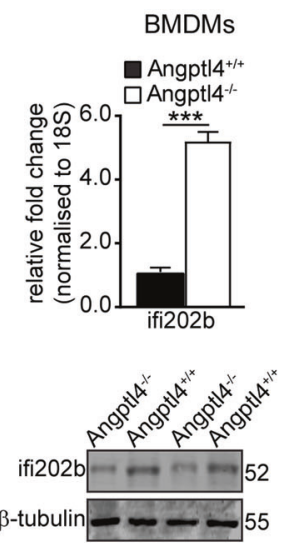

B
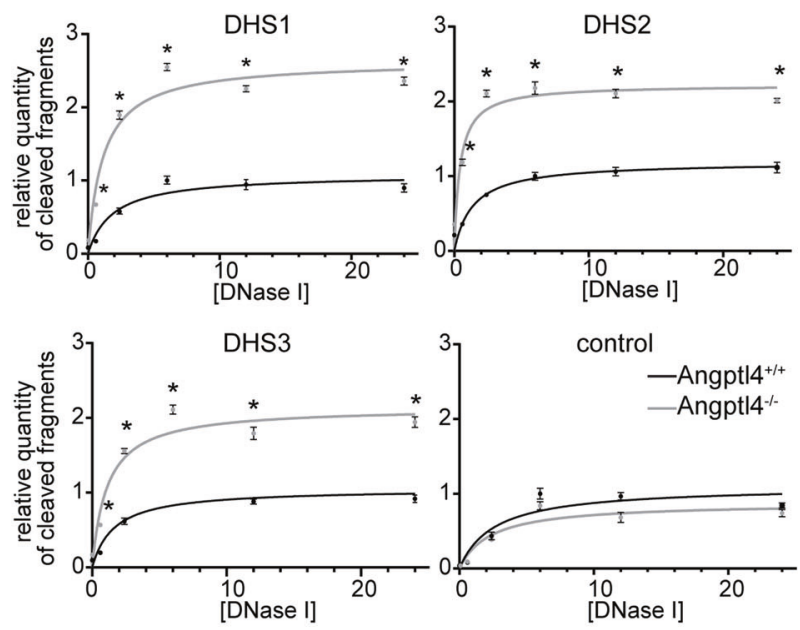

C
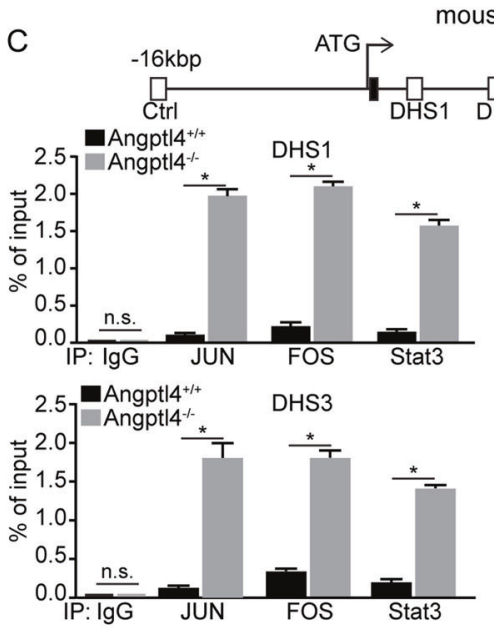

E

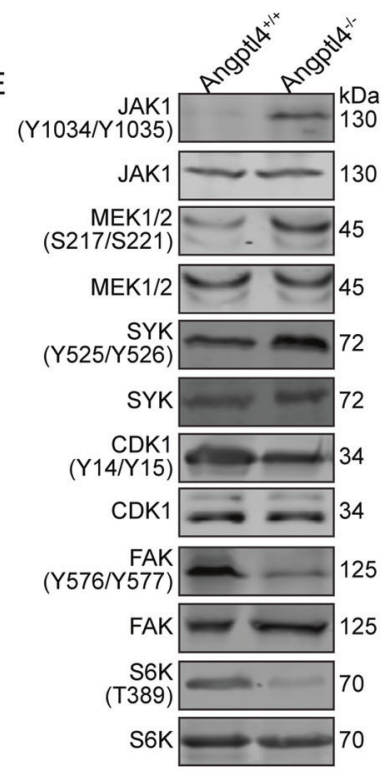

bone marrow were cultured in wound exudate to mimic in vivo differentiation conditions (Fig. 3A, S8A). Regardless of the mouse genotype, Angpt/ $4^{+/+}$wound fluid induced a higher macrophage to Ly6C $C^{\text {int }}$ monocyte ratio than Angpt/4-deficient wound fluid, recapitulated the in vivo Angptl $4^{+/+},{\text {Angpt } 14^{-/-}, \text {Angptl4 }}^{\text {lysM-l- }}$

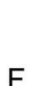
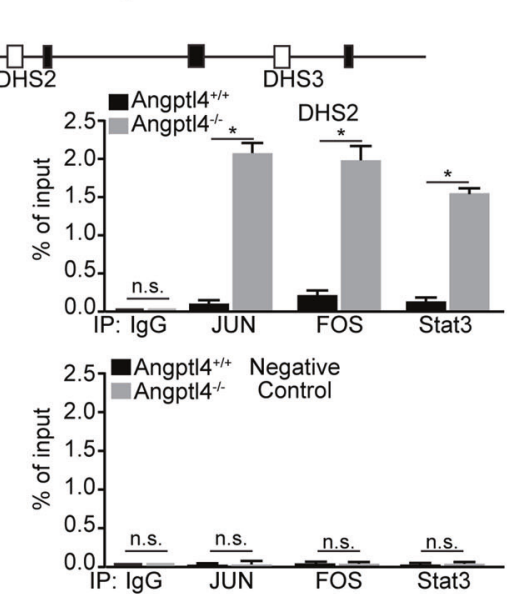

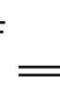

$\mathrm{D}$
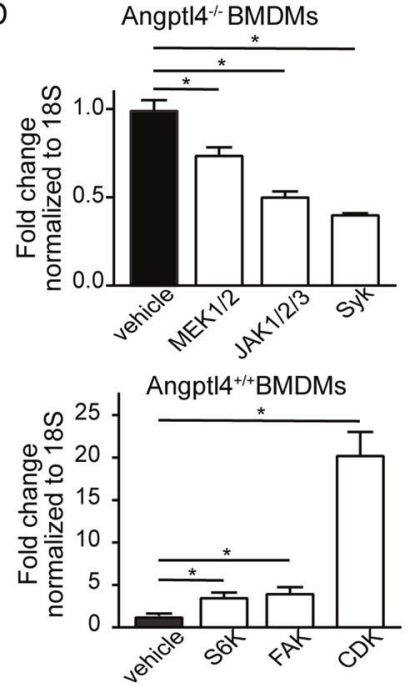
Fig. 2 Signaling network regulating mouse ifi202b in monocytes. A Relative expression of ifi202b mRNA (top) and protein (bottom) in bone marrow-derived monocytes (BMDMs) from Angptl $4^{+/+}$and Angpt $14^{-/-}$mice. B Graphs show the quantification of released DNA fragments at the identified DNAse I hypersensitivity site (DHS) using Angpt $14^{+/+}$and Angptl $4^{-1-}$ BMDMs. A control region that has no known DHS was also quantified as a negative control. C Chromatin immunoprecipitation using antibodies against $p$-Jun, $p$-Fos and $p$-Stat3. ChIP was performed

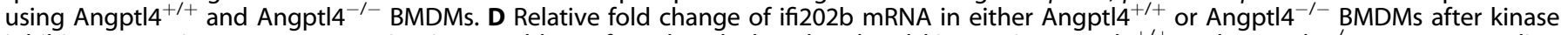
inhibitor screening. E Representative immunoblots of total and phosphorylated kinases in Angpt/4 ${ }^{+/+}$and Angptl4 ${ }^{-1-}$ BMDMs. Loading $^{-1}$ controls were from the same samples. F Connectivity network of the identified kinases involved in the increased (red) or suppressed (blue) expression of ifi202b in monocytes. Blue and red lines indicate the possible networks by which the kinases exert their activating or repressive effects. For panels $\mathbf{A}-\mathbf{D}$, data are from $n=3$ independent experiments with triplicate. ${ }^{*} p<0.05$, ${ }^{* * *} p<0.001$. n.s. not significant.

from Angpt| $4^{-/-}$wounds or Angpt $14^{+/+}$wound fluid treated with anti-cAngptl4 monoclonal antibody (mAb; clone 3F4F5) (Fig. 3C-D). This mAb has been shown previously to block the action of cAngptl4 [24]. However, in Angpt/4 ${ }^{+/+}$wound fluid, the suppression of ifi202b in Angpt/4 ${ }^{-/-}$monocytes did not further induce monocyte differentiation compared with Angptl $4^{-1-}$ monocytes with scrambled siRNA (Fig. 3D). These observations indicate that Angptl 4 and ifi202b act along the same modulatory pathway. Ifi202 modulates the transcriptional activity of several transcription factors, such as NF-KB and AP-1 [25-28], that likely impact multiple gene networks involved in cell fate and functions. Indeed, GSEA and IPA functional analyses revealed that ifi202bassociated canonical pathways and functions involved in the inflammatory response and monocyte cell fate were enriched, such as interleukin, TLR, SMAD, and NOTCH signaling (Fig. 3E, S8C) $[29,30]$. Taken together, our findings clearly highlight that ifi202b, whose expression is regulated by Angpt/4, is a key gatekeeper in monocyte to macrophage differentiation during wound healing.

\section{DISCUSSION}

Angptl4 has been implicated in many inflammatory diseases, such as inflammation-induced lung tissue damage $[24,31]$ and wound healing $[9,16]$. The relationship between Angptl4 and inflammation is context-dependent. However, the precise role of Angpt/4 in immune cells remains unclear. We showed that Angptl 4 in wounds regulates monocyte differentiation to macrophages and coordinates the clearance of neutrophils, thus facilitating the transition from the inflammatory to the proliferative phase of wound healing. There were fewer monocyte-derived macrophages in the absence of Angptl4 in the wound microenvironment, resulting in a prolonged neutrophil presence. The underlying mechanism involves a kinome network that modulates the expression of ifi202b, a transcriptional modulator, to affect multiple pathways involved in cell fate and the inflammatory response.

Macrophage-mediated phagocytosis of apoptotic neutrophils is a key event for transitioning from the inflammatory to the proliferative phase [4, 32]. This dysregulated dynamism between neutrophils and macrophages contributed to the delayed wound healing and stalled inflammation previously observed in Angpt/ $4^{-/-}$mice $[9,16]$. Angpt/4 deficiency in wounds prolongs neutrophil presence and reduces CCR2- and CX3CR1-expressing monocyte-derived macrophages, which are responsible for promoting wound healing [33]. There were fewer mature monocyte-derived macrophages despite similar infiltration of monocytes into Angpt/4 ${ }^{-/-}$wounds than Angptl $4^{+/+}$wounds, as evidenced by flow cytometric and scRNAsequencing analyses. Our analysis also confirmed a decreased ability of Ly6C ${ }^{\text {int }}$ monocytes to acquire an MHC- $I^{+v e}$ phenotype in Angpt $14^{-1-}$ wounds. Consequently, the persistent neutrophil presence and their associated inflammatory response were detrimental to wound repair, which is a major driver of chronic wounds [34].

Angpt/4-deficient wound monocytes and macrophages have elevated expression of ifi202b, which is not observed in other wound immune cells. We revealed that an Angpt/4-associated kinome network involving FAK and Syk, among other kinases, modulates the expression of ifi202b. The activation of FAK by Angpt/4 was consistent with previous studies showing that Angpt/4 interacts with specific integrins to activate integrin-FAK signaling, affecting the cellular response in a context-dependent manner [9, 35]. Syk is a nonreceptor tyrosine kinase highly expressed in hematopoietic cells $[36,37]$. The activation of Syk leads to downstream activation of signaling mediators such as phosphoinositide 3-kinase and Rac/Rho signaling that contribute to immune cell responses [243]. An in vitro reconstituted wound microenvironment study showed that wound fluid derived from Angpt/ $4^{+/+}$wounds could stimulate monocyte to macrophage differentiation, regardless of monocyte genotype. By silencing ifi202b expression, Angpt $14^{-1-}$ monocytes could overcome impaired differentiation due to Angpt/4 deficiency or depletion in wound fluids, resulting in more monocyte-derived macrophages. It is also interesting that Angpt/4 deficiency in macrophages increased the secretion of more TNF-a than wild-type macrophages following LPS stimulation [12]. This observation is consistent with a stalled or enhanced inflammation phase in Angptl4-deficient wounds. Our GSEA of ifi202b-associated pathways revealed that signaling mediators, such as MAP kinase, SMAD, and NOTCH signaling mediators, were enriched, which are also crucial regulators of monocyte to macrophage differentiation [30, 38]. Interestingly, the cell fates of Ly6C monocytes and the resultant inflammation are coordinately regulated by TLR and Notch signaling, both of which are linked to ifi202b [29]. Conceivably, the dynamics between the activating and suppressive signaling networks due to infiltrated monocytes interrogating the wound microenvironment fine-tune the expression of ifi202b and, consequently, the ratio between monocytes and monocyte-derived macrophages in the wounds.

Our current study identified a role for Angpt/4 in the acute inflammatory response to injury. It is conceivable that Angpt/4 also plays a distinct role in adaptive immunity involving $T$ and $B$ cells, which were underrepresented in our skin wounding model. Other chronic inflammatory models, such as atherosclerosis or arthritis, may be more suitable. Of particular interest is systemic lupus erythematosus (SLE). SLE is an autoimmune disease with common clinical manifestations in skin lesions, hyperkeratosis, and scarring [39]. Treatment strategies against SLE generally involve immunosuppression that poorly addresses disease manifestations. It is widely established that genetic predisposition has a key role in susceptibility to SLE [40]. To date, ifi202b is best studied for its role as an SLE susceptibility gene. Interestingly, Angpt/4 has been identified as a candidate biomarker for lupus nephritis in patients with SLE [41, 42]. In patient sera, Angpt/4 expression was significantly lower than that in healthy controls. Clearly, further investigation will be necessary to confirm a role for Angptl4 in SLE.

In conclusion, Angptl4 in the wound milieu regulates the expression of ifi202b, which acts as a gatekeeper facilitating wound monocyte-to-macrophage differentiation during wound healing.

\section{MATERIALS AND METHODS}

Mouse excisional skin wounding

Eight-weeks old wild-type C57BL/6 J male mice (Invivos, Singapore) and Angpt $14^{-1-}$ male mice (Genentech UNQ171, Mutant Mouse Regional Resource Center (MMRRC)) were housed in a specific-pathogen-free environment and 
A
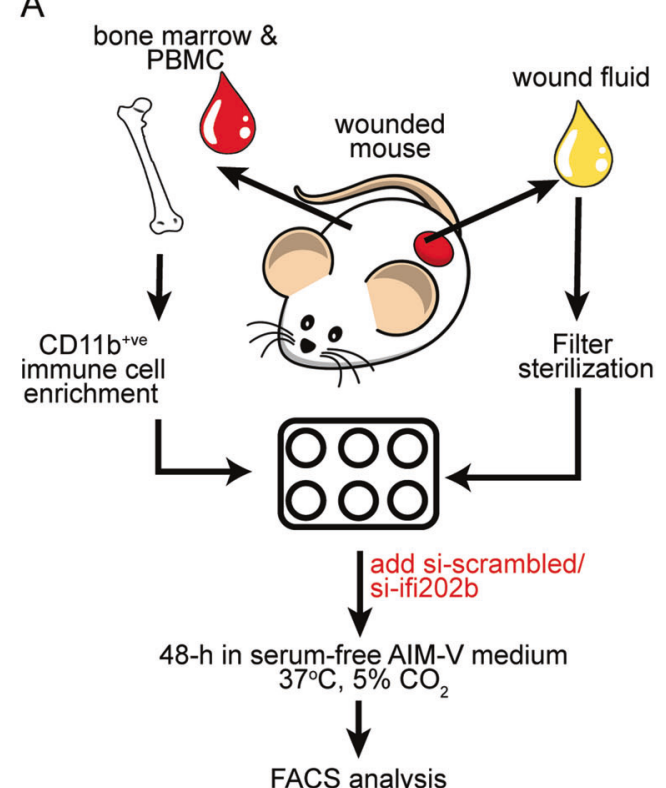

D

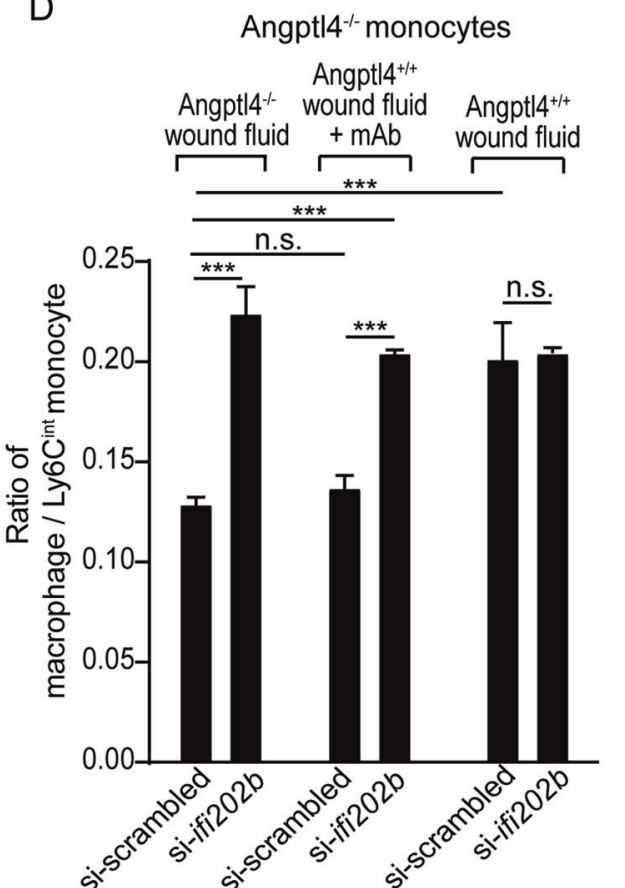

B

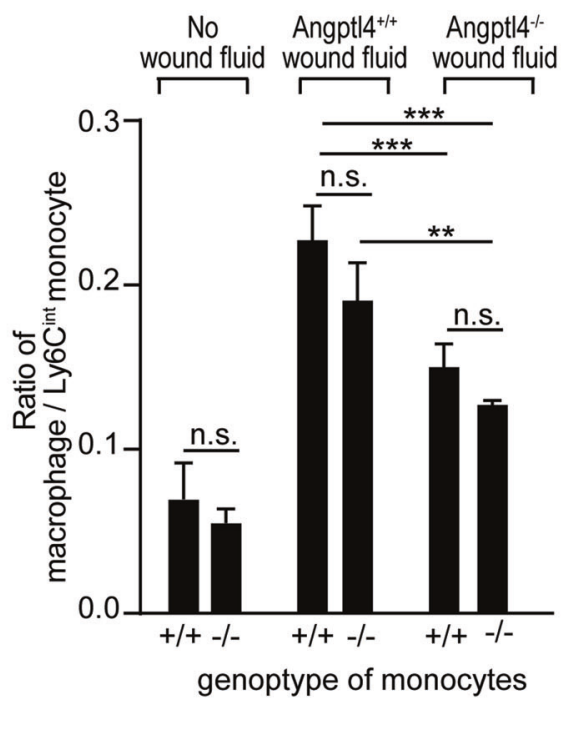

C

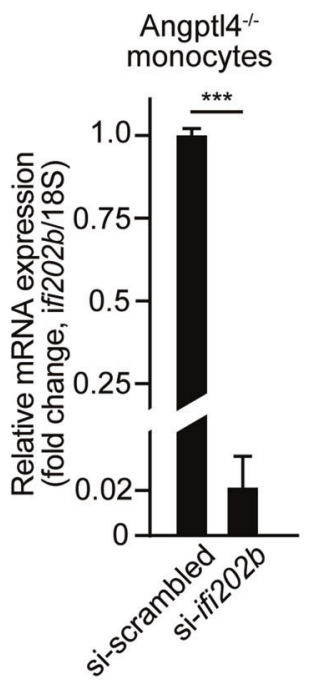

E

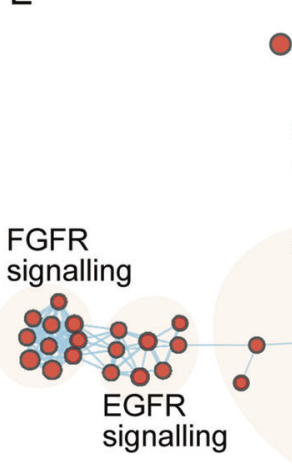

0

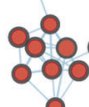

0

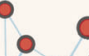

- $\infty$ Interleukin

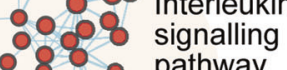

TLR signalling cascade 0

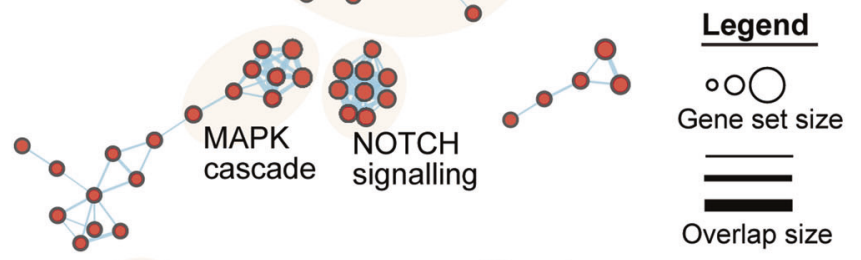

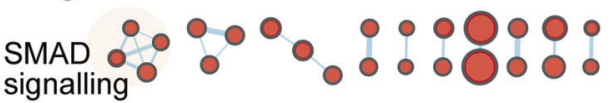

Fig. 3 Ifi202b silencing promotes monocyte differentiation to macrophages. A Schematic illustration of in vitro monocyte culture supplemented with wound exudate to mimic the in vivo wound microenvironment. PBMC, peripheral blood mononuclear cells. B Ratio of macrophage-to-Ly6 $\mathrm{C}^{\mathrm{int}}$ monocytes in Angpt/4 ${ }^{+/+}$and Angpt/4 ${ }^{-/-}$monocytes treated with the indicated wound fluids. Values represent mean \pm SD. C Relative mRNA expression ifi202b in Angpt $14^{-1-}$ monocytes challenged with Angptl4 ${ }^{-1-}$ wound fluid and treated with si-ifi202b. Gene expression was normalized to $18 \mathrm{~S}$ rRNA. D Ratio of macrophage-to-Ly $6 C^{\text {int }}$ monocytes in Angptl $4^{-1-}$ monocytes cultured in the indicated wound fluids. Endogenous expression of ifi202b was suppressed by si-ifi202b. si-scrambled served as negative control. mAb denotes blocking anti-cAngpt/4 monoclonal antibodies. E Functional GSEA of the interaction network associated with ifi202b. Highly enriched cellular functions/processes are highlighted. For panels B-D, data are from $n=3$ independent experiments with triplicate. ${ }^{* *} p<0.01$, ${ }^{* * *} p<0.001$. n.s. not significant.

maintained at $24^{\circ} \mathrm{C}$ and $50 \%$ humidity with a $12 \mathrm{~h}$ light/dark cycle. Under anesthesia, the dorsal skin was depilated and sterilized, and $3 \mathrm{~mm}$ excisional wounds were made with a biopsy punch [43]. Power analysis was used to determined sample size. All experiments were performed following the guidelines of the Institutional Animal Care and Use Committee (NTU-IACUC: A0324, A19034).

\section{In vitro reconstituted wound microenvironment}

CD $11 b^{+}$immune cells isolated from peripheral blood mononuclear cells and bone marrow were cultured in serum-free AIM- $\mathrm{V}^{\mathrm{TM}}$ medium (Gibco) with $2 \%$ penicillin/streptomycin at a density of $2 \times 10^{6}$ cells $/ \mathrm{mL}$ and challenged with $20 \%(\mathrm{v} / \mathrm{v})$ wound fluids from Angpt $14^{+/+}$and Angpt $14^{-1-}$ mice to mimic the wound microenvironment. Angptl4 neutralizing $\mathrm{mAb}$ 
$(10 \mu \mathrm{g} / \mathrm{mL}$; clone $3 \mathrm{~F} 4 \mathrm{F5})$ was added to deplete cAngpt/4 in Angpt/4 ${ }^{+/+}$ wound fluid. After $48 \mathrm{~h}$, cells were retrieved and subjected to FACS analysis using antibodies, as shown in Table S2, based on the gating strategy in Fig. S1A. The macrophage/Ly6 $C^{\text {int }}$ monocyte ratio was calculated based on (Differentiating macrophages [Population 3] + Macrophages [Population 4])/ Ly6C $C^{\text {int }}$ monocytes [Population 2].

\section{Flow cytometry}

Wound tissues were dissociated using $1 \mathrm{mg} / \mathrm{mL}$ Type 3 collagenase and $10 \mathrm{U} / \mathrm{mL}$ DNAsel for $1 \mathrm{~h}$ in a $37^{\circ} \mathrm{C}$ water bath. Cells were separated through a $30 \mu \mathrm{m}$ strainer, blocked with $3 \%$ BSA containing Fc blocker, and stained with selected antibodies (Table S2). FACS data were collected using BD LSRFortessa X-20 and analyzed on FlowJo.

\section{Single-cell RNA sequencing and bioinformatic analysis}

Cell suspensions from wound tissues were stained with CD45-APC and propidium iodide before sorting on a BD FACSAria ${ }^{\mathrm{TM}}$ Fusion. Live immune cells $\left(\mathrm{CD}_{4} 5^{+} \mathrm{PI}^{-}\right)$were collected and washed with PBS before proceeding to single-cell library preparation. Single-cell droplets and cDNA libraries were prepared using a Chromium Single Cell 3' V3 kit (10X Genomics) according to the manufacturer's instructions. The cDNA libraries were sequenced on an Illumina NovaSeq6000. Raw sequencing reads were aligned to the mm10 mouse reference genome using Cell Ranger (v3.1.0) to generate single-cell count matrices that were normalized, integrated, and annotated using Seurat (v3.0) [44].

\section{Statistical analysis}

Statistical analysis was performed using Prism 9 (GraphPad Software Inc). Unless otherwise stated, statistical significance was calculated using unpaired Student's $t$-test for comparisons between two groups or oneway ANOVA followed by Tukey's test for comparisons between multiple $(>2)$ groups. A $p$-value of $<0.05$ was considered statistically significant. Power analysis was used to determined sample size.

\section{DATA AVAILABILITY}

Single-cell RNA-seq data were deposited at the Gene Expression Omnibus (GEO; Accession number: GSE186986). More detailed methods are presented in the Supporting Information.

\section{REFERENCES}

1. Sen CK, Gordillo GM, Roy S, Kirsner R, Lambert L, Hunt TK, et al. Human skin wounds: a major and snowballing threat to public health and the economy. Wound Repair Regen. 2009;17:763-71.

2. Canedo-Dorantes L, Canedo-Ayala M. Skin acute wound healing: a comprehensive review. Int J Inflam. 2019;2019:3706315.

3. Reinke JM, Sorg H. Wound repair and regeneration. Eur Surg Res. 2012;49:35-43.

4. Nathan C. Neutrophils and immunity: challenges and opportunities. Nat Rev Immunol. 2006;6:173-82.

5. Eming SA, Krieg T, Davidson JM. Inflammation in wound repair: molecular and cellular mechanisms. J Invest Dermatol. 2007;127:514-25.

6. Kyriakides TR, Bornstein P. Matricellular proteins as modulators of wound healing and the foreign body response. Thromb Haemost. 2003;90:986-92.

7. Bornstein P. Matricellular proteins: an overview. J Cell Commun Signal. 2009;3:163-5.

8. Dijk W, Kersten S. Regulation of lipoprotein lipase by Angpt/4. Trends Endocrinol Metab. 2014;25:146-55.

9. Goh YY, Pal M, Chong HC, Zhu P, Tan MJ, Punugu L, et al. Angiopoietin-like 4 interacts with integrins beta1 and beta5 to modulate keratinocyte migration. Am J Pathol. 2010;177:2791-803.

10. Huang RL, Teo Z, Chong HC, Zhu P, Tan MJ, Tan CK, et al. ANGPTL4 modulates vascular junction integrity by integrin signaling and disruption of intercellular VEcadherin and claudin-5 clusters. Blood 2011;118:3990-4002.

11. Aryal B, Rotllan N, Araldi E, Ramirez CM, He S, Chousterman BG, et al. ANGPTL4 deficiency in haematopoietic cells promotes monocyte expansion and atherosclerosis progression. Nat Commun. 2016;7:12313.

12. Ding S, Wu D, Lu Q, Qian L, Ding Y, Liu G, et al. Angiopoietin-like 4 deficiency upregulates macrophage function through the dysregulation of cell-intrinsic fatty acid metabolism. Am J Cancer Res. 2020;10:595-609.

13. Suzuki T, Ishii S, Shinohara M, Kawano Y, Wakahashi K, Kawano H, et al. Mobilization efficiency is critically regulated by fat via marrow PPARdelta. Haematologica. 2021;106:1671-83.
14. Zhu P, Goh YY, Chin HF, Kersten S, Tan NS. Angiopoietin-like 4: a decade of research. Biosci Rep. 2012;32:211-9.

15. Teo Z, Chan JSK, Chong HC, Sng MK, Choo CC, Phua GZM, et al. Angiopoietin-like 4 induces a beta-catenin-mediated upregulation of ID3 in fibroblasts to reduce scar collagen expression. Sci Rep. 2017;7:6303.

16. Chong HC, Chan JS, Goh CQ, Gounko NV, Luo B, Wang X, et al. Angiopoietin-like 4 stimulates STAT3-mediated iNOS expression and enhances angiogenesis to accelerate wound healing in diabetic mice. Mol Ther. 2014;22:1593-604.

17. Shay T, Kang J. Immunological Genome Project and systems immunology. Trends Immunol 2013;34:602-9.

18. Guilliams M, Ginhoux F, Jakubzick C, Naik SH, Onai N, Schraml BU, et al. Dendritic cells, monocytes and macrophages: a unified nomenclature based on ontogeny. Nat Rev Immunol. 2014;14:571-8.

19. Trapnell C, Cacchiarelli D, Grimsby J, Pokharel P, Li S, Morse M, et al. The dynamics and regulators of cell fate decisions are revealed by pseudotemporal ordering of single cells. Nat Biotechnol. 2014;32:381-6.

20. Lech $M$, Grobmayr $R$, Weidenbusch $M$, Anders HJ. Tissues use resident dendritic cells and macrophages to maintain homeostasis and to regain homeostasis upon tissue injury: the immunoregulatory role of changing tissue environments. Mediators Inflamm. 2012;2012:951390.

21. Lucas T, Waisman A, Ranjan R, Roes J, Krieg T, Muller W, et al. Differential roles of macrophages in diverse phases of skin repair. J Immunol. 2010;184:3964-77.

22. Consortium EP. An integrated encyclopedia of DNA elements in the human genome. Nature 2012;489:57-74.

23. Schultz GS, Davidson JM, Kirsner RS, Bornstein P, Herman IM. Dynamic reciprocity in the wound microenvironment. Wound Repair Regen. 2011;19:134-48.

24. Li L, Foo BJW, Kwok KW, Sakamoto N, Mukae H, Izumikawa K, et al. Antibody treatment against angiopoietin-like 4 reduces pulmonary edema and injury in secondary pneumococcal pneumonia. mBio. 2019.10:e02469, https://doi.org/ 10.1128/mBio.02469-18.

25. Panchanathan $\mathrm{R}, \mathrm{Xin} \mathrm{H}$, Choubey D. Disruption of mutually negative regulatory feedback loop between interferon-inducible p202 protein and the E2F family of transcription factors in lupus-prone mice. J Immunol. 2008;180:5927-34.

26. Ma $X Y$, Wang $H$, Ding $B$, Zhong $H$, Ghosh $S$, Lengyel $P$. The interferon-inducible p202a protein modulates NF-kappaB activity by inhibiting the binding to DNA of p50/p65 heterodimers and p65 homodimers while enhancing the binding of p50 homodimers. J Biol Chem. 2003;278:23008-19.

27. Min W, Ghosh S, Lengyel P. The interferon-inducible $\mathrm{p} 202$ protein as a modulator of transcription: inhibition of NF-kappa B, c-Fos, and c-Jun activities. Mol Cell Biol. 1996;16:359-68.

28. Rozzo SJ, Allard JD, Choubey D, Vyse TJ, Izui S, Peltz G, et al. Evidence for an interferon-inducible gene, Ifi202, in the susceptibility to systemic lupus. Immunity 2001;15:435-43.

29. Gamrekelashvili J, Kapanadze T, Sablotny S, Ratiu C, Dastagir K, Lochner M, et al. Notch and TLR signaling coordinate monocyte cell fate and inflammation. Elife. 2020;9:e57007.

30. Bhattacharya A, Ghosh P, Prasad R, Ghosh A, Das K, Roy A, et al. MAP Kinase driven actomyosin rearrangement is a crucial regulator of monocyte to macrophage differentiation. Cell Signal. 2020;73:109691.

31. Guo L, Li S, Zhao Y, Qian P, Ji F, Qian L, et al. Silencing Angiopoietin-Like Protein 4 (ANGPTL4) protects against lipopolysaccharide-induced acute lung injury via regulating SIRT1 /NF-kB pathway. J Cell Physiol. 2015;230:2390-402.

32. Widgerow AD. Cellular resolution of inflammation-catabasis. Wound Repair Regen. 2012;20:2-7.

33. Rodero MP, Hodgson SS, Hollier B, Combadiere C, Khosrotehrani K. Reduced II17a expression distinguishes a $\mathrm{Ly} 6 \mathrm{C}(\mathrm{lo}) \mathrm{MHCll}(\mathrm{hi})$ macrophage population promoting wound healing. J Invest Dermatol. 2013;133:783-92.

34. Wilgus TA, Roy S, McDaniel JC. Neutrophils and wound repair: positive actions and negative reactions. Adv Wound Care (N. Rochelle). 2013;2:379-88.

35. Zhu P, Tan MJ, Huang RL, Tan CK, Chong HC, Pal M, et al. Angiopoietin-like 4 protein elevates the prosurvival intracellular $\mathrm{O} 2(-): \mathrm{H}_{2} \mathrm{O}_{2}$ ratio and confers anoikis resistance to tumors. Cancer Cell 2011;19:401-15.

36. Mocsai A, Ruland J, Tybulewicz VL. The SYK tyrosine kinase: a crucial player in diverse biological functions. Nat Rev Immunol. 2010;10:387-402.

37. Yanagi S, Inatome R, Takano T, Yamamura H. Syk expression and novel function in a wide variety of tissues. Biochem Biophys Res Commun. 2001;288:495-8.

38. Kurotaki D, Sasaki H, Tamura T. Transcriptional control of monocyte and macrophage development. Int Immunol. 2017;29:97-107.

39. Kuhn A, Bonsmann G, Anders HJ, Herzer P, Tenbrock K, Schneider M. The diagnosis and treatment of systemic lupus erythematosus. Dtsch Arztebl Int. 2015;112:423-32.

40. Fairhurst $A M$, Wandstrat $A E$, Wakeland EK. Systemic lupus erythematosus: multiple immunological phenotypes in a complex genetic disease. Adv Immunol. 2006;92:1-69. 
41. Vanarsa K, Soomro S, Zhang T, Strachan B, Pedroza C, Nidhi M, et al. Quantitative planar array screen of 1000 proteins uncovers novel urinary protein biomarkers of lupus nephritis. Ann Rheum Dis. 2020;79:1349-61.

42. Hutcheson J, Ye Y, Han J, Arriens C, Saxena R, Li QZ, et al. Resistin as a potential marker of renal disease in lupus nephritis. Clin Exp Immunol. 2015;179:435-43.

43. Tan NS, Wahli W. Studying wound repair in the mouse. Curr Protoc Mouse Biol. 2013;3:171-85.

44. Hao Y, Hao S, Andersen-Nissen E, Mauck WM 3rd, Zheng S, Butler A, et al. Integrated analysis of multimodal single-cell data. Cell 2021;184:3573-87.

\section{ACKNOWLEDGEMENTS}

This research is supported by the Nanyang Technological University Singapore StartUp Grant (\#001325-00001) to NST. Single-cell RNA sequencing of the wound healing immune landscape was supported by a 10x Genomics Grant awarded to NST. The computational work in this manuscript was performed using resources from the National Supercomputing Centre Singapore.

\section{AUTHOR CONTRIBUTIONS}

WKJW, ZSL, CKO, BPH, ZGRL, YSY, MIGV, WWRT, and HSC performed experiments. YSY and MIGV provided key reagents and specimens. WKJW, ZSL, and HSC performed bioinformatics analyses. WKJW, ZSL, HSC, and NST designed the research, interpreted the data, and wrote the paper.

\section{COMPETING INTERESTS}

The authors declare no competing interests.

\section{ADDITIONAL INFORMATION}

Supplementary information The online version contains supplementary material available at https://doi.org/10.1038/s41419-022-04638-7.

Correspondence and requests for materials should be addressed to Nguan Soon Tan.

Reprints and permission information is available at http://www.nature.com/reprints

Publisher's note Springer Nature remains neutral with regard to jurisdictional claims in published maps and institutional affiliations.

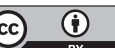

Open Access This article is licensed under a Creative Commons Attribution 4.0 International License, which permits use, sharing, adaptation, distribution and reproduction in any medium or format, as long as you give appropriate credit to the original author(s) and the source, provide a link to the Creative Commons license, and indicate if changes were made. The images or other third party material in this article are included in the article's Creative Commons license, unless indicated otherwise in a credit line to the material. If material is not included in the article's Creative Commons license and your intended use is not permitted by statutory regulation or exceeds the permitted use, you will need to obtain permission directly from the copyright holder. To view a copy of this license, visit http://creativecommons. org/licenses/by/4.0/.

(c) The Author(s) 2022 